\title{
Nondegrading photoluminescence in porous silicon by deuterium plasma treatment
}

\author{
C. H. Chen and Y. F. Chen \\ Department of Physics, National Taiwan University, Taipei, Taiwan, Republic of China
}

An Shih and S. C. Lee

Department of Electrical Engineering, National Taiwan University, Taipei, Taiwan, Republic of China

(Received 21 July 2000; revised manuscript received 3 July 2001; published 26 April 2002)

\begin{abstract}
Porous silicon (PS) with nondegrading photoluminescence (PL) was obtained by deuterium plasma treatment. We demonstrate that the structural stability of deuterated PS is much better than that of normal PS. The secondary-ion-mass spectrometry and infrared-absorption spectra reveal the formation of Si-D bonds on the surface of PS. The Raman spectra show the evidence of the coupling between the Si-D wagging mode and transverse Si-Si optical phonon. This coupling leads to the relaxation of the accumulated energy and hence the reduction of the bond breaking. All our results indicate that the possible mechanism of the PL stability in deuterated PS can be attributed to the existence of Si-D bonds on the surface of PS.
\end{abstract}

DOI: 10.1103/PhysRevB.65.195307

PACS number(s): $81.40 . \mathrm{Tv}, 78.55 . \mathrm{Hx}$

\section{INTRODUCTION}

Since porous silicon (PS) shows strong photoluminescence (PL) in the visible range at room temperature, it has been investigated intensively over the past ten years. ${ }^{1-4}$ Recently, most of the research has been directed to clarify the luminescence mechanism ${ }^{1,5-9}$ and fabricate a PS-based light emitter. ${ }^{10-12}$ However, the problem of the degradation ${ }^{13-15}$ and the relatively low efficiency ${ }^{16}$ of PL remain unresolved. The PL degradation with a blueshift or redshift of the peak position of the freshly made samples is believed to originate from the chemical instability of the porous silicon surface. ${ }^{14,15,17-19}$ Due to the activity of oxygen, the Si-H bonds on the surfaces of the Si nanocrystallite will be broken and the number of the Si dangling bonds will increase. Because the dangling bond is a very efficient nonradiative recombination center, this process will ultimately lead to the degradation of the PL intensity. It is quite interesting to note that the similar property of photodegradation has also been observed in hydrogenated amorphous-silicon alloy $\left(a\right.$-Si:H). ${ }^{20}$ Recently, hot-electron degradation in silicon metal-oxide-semiconductor field-effect transistors (MOSFET's) has been found to be greatly reduced by passivating the silicon-silicon dioxide interface using deuterium instead of hydrogen..$^{21}$ And several reports ${ }^{22-25}$ also showed that the incorporation of deuterium instead of hydrogen in amorphous silicon tends to slow the decay of its photoconductivity under illumination. This suggests that the incorporation of deuterium may be used to solve the degradation problem in porous silicon. Indeed, in this paper, we demonstrated that the stability of porous silicon can be greatly improved after deuterium treatment. We showed that the underlying mechanism of the improved stability could be attributed to the coupling between the Si-D wagging mode and transverse $\mathrm{Si}-\mathrm{Si}$ optical phonon mode. Our result should be beneficial for the application of porous silicon.

\section{EXPERIMENT}

Porous silicon samples were prepared by anodizing $\mathrm{Si}$ wafers. The silicon wafers are $p$-type $B$ doped with resistance of
$8-11 \Omega \mathrm{cm}$ and $(1,0,0)$ orientation, which were immerged in a solution of HF: ethanol $=1: 1$ at a current density of 10 $\mathrm{mA} / \mathrm{cm}^{2}$ for $20-60 \mathrm{~min}$. The diameter of the macropore is about $1 \mu \mathrm{m}$ and a depth of $100 \mu \mathrm{m}$. We obtain the macroporous layers with a porosity of about $80 \%$. To improve the surface stability, the porous silicon films were treated by $\mathrm{D}_{2}$ plasma. The condition for the $\mathrm{D}_{2}$ plasma treatment were as follows: substrate temperature, $250^{\circ} \mathrm{C}$, where the substrate is heated by a slot heater embedded in a piece of copper; chamber pressure, 6 torr; and the $\mathrm{D}_{2}$ gas flow rate, 3 SCCM (SCCM denotes cubic centimeter per minute at STP). The power and duration of the $\mathrm{D}_{2}$ plasma treatment are about $9 \mathrm{~W}$ and $90 \mathrm{~min}$, respectively. The condition of $\mathrm{D}_{2}$ plasma treatment has been optimized. In order to find the optimized condition, many parameters must be considered, such as gas flow rate, chamber pressure, input power, electrode spacing, temperature and time, etc. According to the optimized condition, the surface of the sample will not be etched by deuterium plasma. We named the treated sample as deuterated PS. The room-temperature PL spectra were recorded by a SPEX $0.85-\mathrm{m}$ double spectrometer, and a photomultiple tube. A He-Cd laser working at $325 \mathrm{~nm}$ was used as the excitation source and the power density is about 0.4 $\mathrm{W} / \mathrm{cm}^{2}$. The infrared spectra were measured using a Nicolet Magna IR model 550 Fourier transform infrared spectrometer at room temperature. The Raman signal was measured by a DILOR XY800 triple-grating Raman spectrometer. The Raman spectra of the samples were measured at room temperature in vacuum using an Ar laser with a 514.5-nm wavelength and the power density is about $3 \mathrm{~W} / \mathrm{cm}^{2}$. Secondaryion-mass spectroscopy (SIMS) was also used to measure the deuterium contents of deuterated PS. For the SIMS measurements, $\mathrm{Cs}^{+}$ions were used to bombard the sample and the secondary ions were accelerated to a mass spectrometry for separating atoms or molecules with different masses. The advantage of using $\mathrm{Cs}^{+}$ion is to enhance the secondary-ion yield and increase the sensitivity.

\section{RESULTS AND DISCUSSION}

Figure 1 shows the PL spectra of deuterated PS and normal PS. The PL spectra of normal PS exhibit a broad peak 


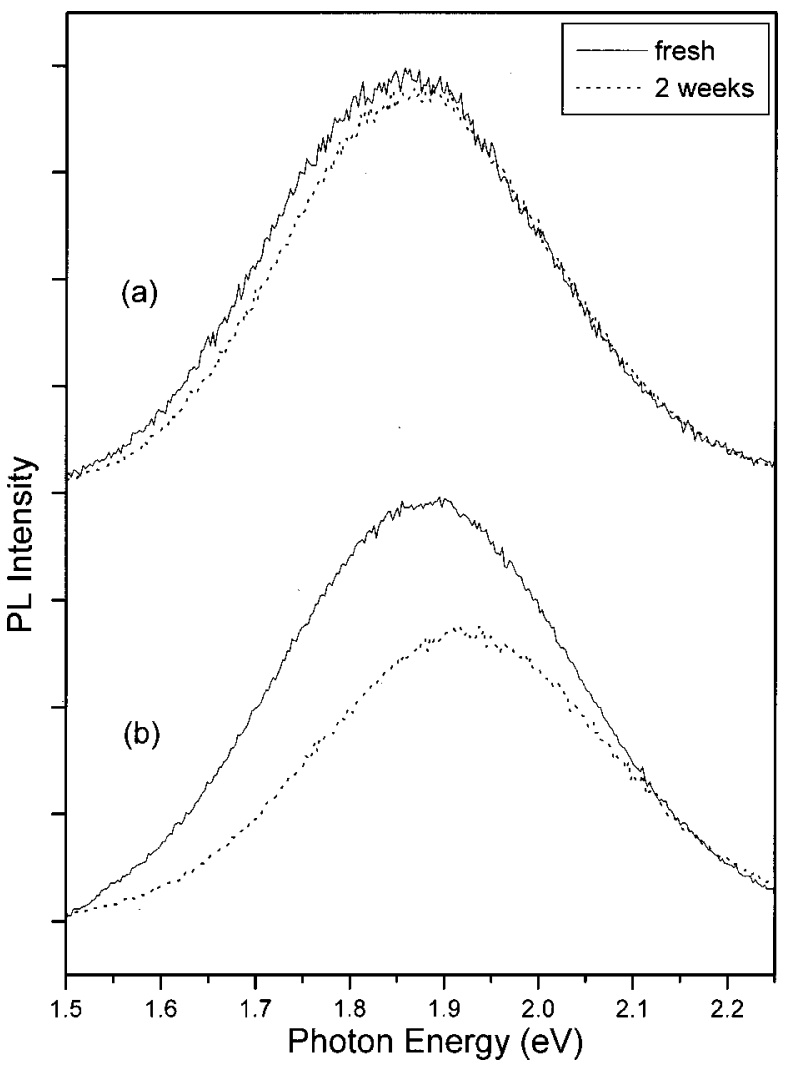

FIG. 1. The time evolution of the PL spectra for (a) the deuterated PS sample and (b) normal PS exposed to ambient air at room temperature.

centered at about $1.9 \mathrm{eV}$. This is a typical result for the porous silicon prepared by the similar condition. ${ }^{1,26,27}$ After deuterium plasma treatment, the PL spectra of deuterated PS are very similar to that of normal PS. It indicates that the 1.9-eV PL spectrum of porous silicon does not change when the surface complexes are altered. The detailed studies of the surface complexes will be shown below. With time evolution for the samples exposed to ambient air at room temperature, the PL intensity of normal PS degrades quickly and the peak position blueshifts as shown in Fig. 1(b). Many previous reports ${ }^{14,15,17}$ also obtained the same result for as-grown PS. It is worth noting that under different preparation conditions and environment, a redshift has also been reported. ${ }^{18,19} \mathrm{Nev-}$ ertheless, the PL intensity of deuterated porous silicon decays with time extremely slowly and the peak energy nearly remains at the same position as shown in Fig. 1(a). This result provides evidence showing that the structural stability of deuterated PS is better than that of normal PS.

In many reports, ${ }^{28-30}$ the PL degradation with a blueshift of the peak position subsequent to the normal preparation is believed to originate from the chemical instability of the PS surface. Under the attack of oxygen, the Si-H bonds on the surfaces of the Si macrocrystallites will be broken and the quantity of the Si dangling bonds will increase. The variation will finally result in the degradation of PL intensity. The oxidation of PS in the degraded sample was confirmed by the growth of the Si-O-Si vibrational mode at $1100 \mathrm{~cm}^{-1}$. In Fig. 2(a), we show the time evolution of the infrared spectra of
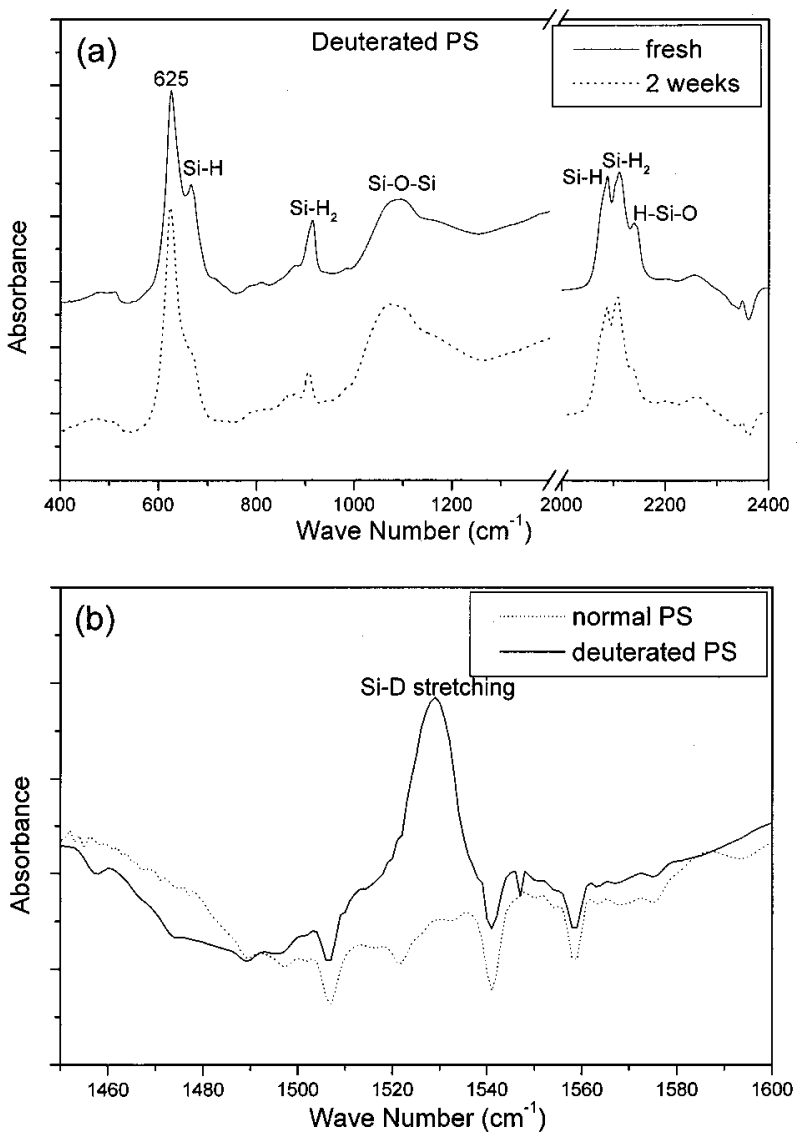

FIG. 2. (a) Fourier transform infrared-absorption spectra of deuterated porous silicon. It shows that the intensity of the Si-O-Si vibrational mode does not increase when the deuterated sample is exposed to air. (b) Fourier transform infrared-absorption spectra of deuterated and normal porous silicon.

deuterated PS. Unlike that of normal PS, the intensity of the vibrational mode at $1100 \mathrm{~cm}^{-1}$ for deuterated PS does not change. This result also supports the fact that the stability of deuterated PS is better than that of normal PS.

It is known that due to its unstable structure, the structure of porous silicon under illumination is subject to significant evolution with time, which directly affects its luminescent properties. ${ }^{13-15,26,27}$ This behavior is quite true for normal porous silicon as shown in Fig. 3. We can see that the PL intensity of normal PS suffers a significant degradation under illumination. Nevertheless, under the same condition, the PL intensity of deuterated porous silicon decays much slower than that of normal PS as shown in Fig. 3. This result implies that the structure of deuterated PS is more resistant to external illumination. A further test of the stability of deuterated PS was conducted under an external bias. In Fig. 4, we can clearly see that under the same condition, the PL intensity of the normal PS sample is reduced by about $65 \%$ when bias is about $40 \mathrm{v}$, while that of deuterated PS decreases only by about $15 \%$. Together with the above experiment of the exposure to ambient air at room temperature, we therefore conclude that the structural stability of deuterated PS is much better than that of normal PS. In order to confirm that the improved stability described above is due to the effect of 




FIG. 3. The normalized PL decay intensity as a function of illumination time for deuterated and normal porous silicon.

deuteration. The hydrogen plasma treatment has also been performed for the freshly made PS as well as amorphous silicon. We found that the stability for both materials does not have any improvement.

To explore the origin of the different stability of PL spectra between deuterated PS and normal PS, we measured secondary-ion-mass spectrometry and infrared-absorption spectra as shown in Figs. 5 and 2(b), respectively. In the SIMS profile, we clearly observed the existence of deuterium atoms in PS after deuterium plasma treatment. Comparing with deuterated and normal SIMS profiles, the deuterium plasma treatment is to produce deuterium radicals which enter the voids of the film to passivate the dangling bonds, and to break and passivate weak bonds. In Fig. 2(b), the deuterated PS film clearly displays the $1530-\mathrm{cm}^{-1}$ peak for Si-D stretching, while normal porous silicon film does not contain any Si-D related absorbance peak. Evidently, our results clearly show that deuterium can be successfully incorporated into PS after deuterium plasma treatment. It is generally believed that the degradation of normal PS is due to the chemical instability of the PS surface. Under external perturbation, the weak bonds on the PS surface, such as $\mathrm{Si}-\mathrm{H}$ and $\mathrm{Si}-\mathrm{O}_{2}$ complexes, will be broken and the number of $\mathrm{Si}$ dangling bonds is enhanced. Because the dangling bond is a very efficient nonradiative recombination center, the optical property of the films therefore degrades. This phenomenon is similar to the well-known Staebler-Wronski effect in hydro-

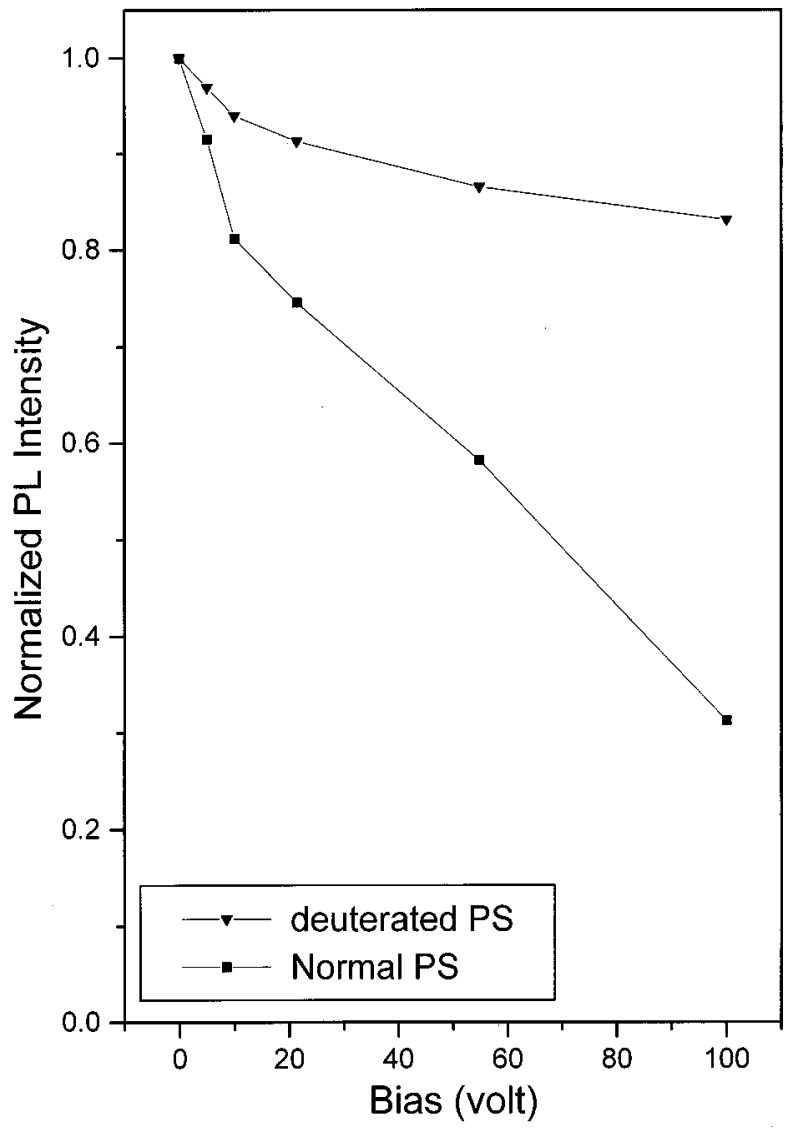

FIG. 4. The drop of the PL peak intensity versus applied lateral voltage for deuterated and normal porous silicon.

genated amorphous silicon. ${ }^{20}$ According to the recent reports ${ }^{2-24}$ of hydrogenated and deuterated amorphous silicon $(a-\mathrm{Si}: \mathrm{H}$ and $a-\mathrm{Si}: \mathrm{D})$, the incorporation of deuterium instead of hydrogen in amorphous silicon tends to slow the decay of its photoconductivity under illumination. The authors $^{23,29,30}$ suggested that the improvement of the degradation results from the coupling between the $\mathrm{Si}$-Si lattice transverse-optical (TO) mode and the Si-D wagging mode, because the $\mathrm{Si}-\mathrm{D}$ wagging mode is very close to the $\mathrm{Si}-\mathrm{Si} \mathrm{TO}$ mode, and the accumulated energy on Si-D sites can be eas-
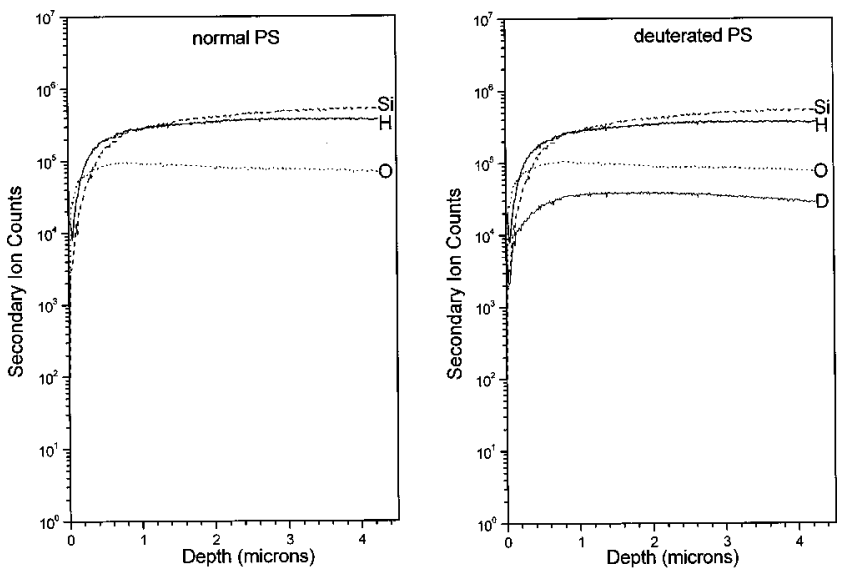

FIG. 5. SIMS profile for deuterated and normal PS. 


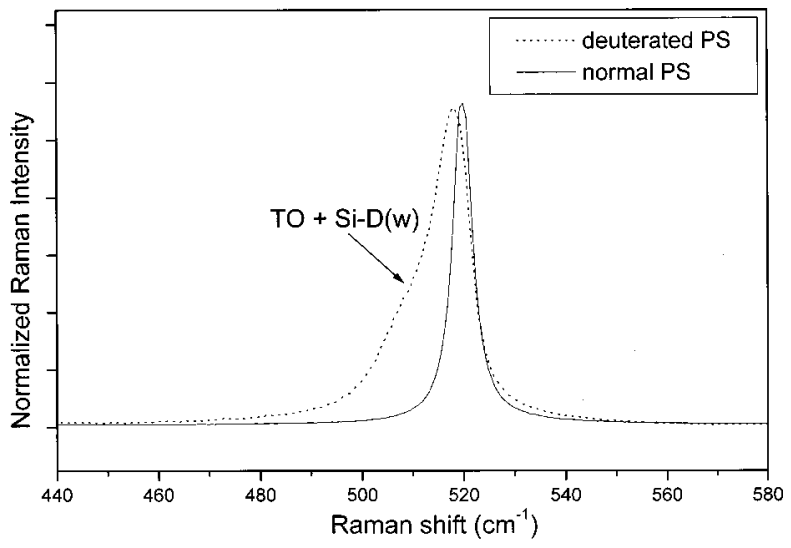

FIG. 6. Raman spectra of normal PS and deuterated PS.

ily released. Therefore the probability of breaking the nearby weak bonds is significantly reduced. Based on the same reason, the existence of Si-D bonds on the porous silicon surface can also lead to the improvement of the structural stability of porous silicon.

In order to verify the above argument, i.e., the coupling between the Si-Si TO mode and the Si-D wagging mode, we perform the Raman spectra of normal PS and deuterated PS as shown in Fig. 6. It is well known ${ }^{33}$ that the Raman spectrum of the Si-Si longitudinal-optical (LO) mode locates at $522 \mathrm{~cm}^{-1}$. In Fig. 6, we can clearly see that the Raman spectrum of normal PS has a peak at $519 \mathrm{~cm}^{-1}$ with a full width at half maximum of $5 \mathrm{~cm}^{-1}$. The reason for the redshift of the Raman peak of normal PS can be easily understood by the effect of the quantum confinement of the optical phonon. ${ }^{33,34}$ As shown in Fig. 6, in addition to the peak at $518 \mathrm{~cm}^{-1}$, the Raman spectrum of deuterated PS has a shoulder around $509 \mathrm{~cm}^{-1}$, which is due to the existence of the $\mathrm{Si}-\mathrm{D}$ wagging mode. Since the Si-D wagging mode is very close to the Si-Si TO phonon of PS, our measurement thus provides a firm evidence to show the coupling between the $\mathrm{Si}-\mathrm{Si} \mathrm{TO}$ phonon and $\mathrm{Si}-\mathrm{D}$ wagging mode. It is worth noting that the evidence of the coupling between the Si-D wagging mode and the Si-Si TO phonon mode shown here is much clearer than in previous reports. ${ }^{31,32}$

\section{CONCLUSION}

In conclusion, we report that porous silicon with stable and strong red emission can be obtained by deuterium plasma treatment. We demonstrated that the structural stability of deuterated PS is much better than that of normal PS. Spectroscopic studies for the deuterated porous silicon exposed to ambient air show that the room-temperature PL intensity does not degrade and the peak position remains unchanged. The secondary-ion-mass spectrometry and Fourier transform infrared-absorption spectra clearly indicate that the deuterated PS contains Si-D bonds. Similar to the studies on amorphous silicon, the improved stability can be attributed to the coupling between the Si-Si TO mode and the Si-D wagging mode. Because of the coupling, the accumulated energy can be easily relaxed and the probability of bond breaking is reduced. Our Raman spectra provide a firm evidence to show the coupling between the Si-Si TO phonon and the Si-D wagging mode. We therefore offer a convenient method to obtain porous silicon with stable and strong luminescence, and provide the underlying mechanism for the improved stability. We believe that our result is very useful for the application of porous silicon.

\section{ACKNOWLEDGMENT}

This work was partly supported by the National Science Council of the Republic of China.
${ }^{1}$ L. T. Canham, Appl. Phys. Lett. 57, 1046 (1990).

${ }^{2}$ A. Bsiesy, J. C. Vial, F. Gaspard, R. Herino, M. Ligeon, F. Muller, R. Romestain, A. Wasiela, A. Halimaoui, and G. Bomchil, Surf. Sci. 254, 195 (1991).

${ }^{3}$ N. Koshida and H. Koyama, Jpn. J. Appl. Phys., Part 2 30, L1221 (1991)

${ }^{4}$ K. J. Lockwood, Solid State Commun. 92, 101 (1994).

${ }^{5}$ A. G. Cullis and L. T. Canham, Nature (London) 353, 335 (1991).

${ }^{6}$ F. Buda, J. Kohanoff, and M. Parrinello, Phys. Rev. Lett. 69, 1272 (1992).

${ }^{7}$ I. M. Chang and Y. F. Chen, J. Appl. Phys. 82, 3514 (1997).

${ }^{8}$ G. G. Qin and Y. Q. Jia, Solid State Commun. 86, 559 (1993).

${ }^{9}$ Will H. Green, Eric J. Lee, Jeffrey M. Lauerhaas, Theodore W. Bitner, and Michael J. Sailor, Appl. Phys. Lett. 67, 1468 (1995).

${ }^{10}$ A. Loni, A. J. Simons, T. I. Cox, P. D. J. Calcott, and L. T. Canham, Electron. Lett. 31, 1288 (1995).

${ }^{11}$ L. Tsybeskov, S. P. Duttagupta, K. D. Hirschman, and P. M. Fauchet, Appl. Phys. Lett. 68, 2058 (1996).

${ }^{12}$ K. D. Hirschman, L. Tsybeskov, S. P. Duttagupta, and P. M.
Fauchet, Nature (London) 384, 338 (1996).

${ }^{13}$ D. W. Cooke, B. L. Bennett, E. H. Farnum, W. L. Hults, K. E. Sickafus, J. F. Smith, T. N. Taylor, and P. Tiwari, Appl. Phys. Lett. 68, 1663 (1996).

${ }^{14}$ L. T. Canham, M. R. Houlton, W. Y. Leong, C. Pickering, and J. M. Keen, J. Appl. Phys. 70, 422 (1991).

${ }^{15}$ M. A. Tischler, R. T. Collins, J. H. Stathis, and J. C. Tsang, Appl. Phys. Lett. 60, 639 (1992).

${ }^{16}$ H. Koyama, T. Nakagawa, T. Ozaki, and N. Koshida, Appl. Phys. Lett. 65, 1656 (1994).

${ }^{17}$ Y. H. Zhang, X. J. Li, L. Zheng, and Q. W. Chen, Phys. Rev. Lett. 81, 1710 (1998).

${ }^{18}$ T. Matsumoto, T. Futagi, H. Mimura, and Y. Kanemitsu, Phys. Rev. B 47, 13876 (1993).

${ }^{19}$ Weimin Zhou, H. Shen, J. F. Harvey, R. A. Lux, M. Dutta, F. Lu, C. H. Perry, R. Tsu, N. M. Kalkhoran, and F. Namavar, Appl. Phys. Lett. 61, 1435 (1992).

${ }^{20}$ K. L. Staebler and C. R. Wronski, J. Appl. Phys. 31, 291 (1977).

${ }^{21}$ J. W. Lyding, K. Hess, and I. C. Kiziyalli, Appl. Phys. Lett. 68, 2526 (1996). 
${ }^{22}$ G. Ganguly, A. Suzuki, S. Yamasaki, K. Nomoto, and A. Matsuda, J. Appl. Phys. 68, 3738 (1990).

${ }^{23}$ W. A. Nevin, H. Yamagushi, K. Asaoka, H. Nishio, and Y. Tawada, Appl. Phys. Lett. 59, 3294 (1991).

${ }^{24}$ C. G. Van de Walle and W. B. Jackson, Appl. Phys. Lett. 69, 2441 (1996).

${ }^{25}$ J. H. Wei, M. S. Sun, and S. C. Lee, Appl. Phys. Lett. 71, 1498 (1997).

${ }^{26}$ C. H. Chen and Y. F. Chen, Solid State Commun. 111, 681 (1999).

${ }^{27}$ C. H. Chen and Y. F. Chen, Appl. Phys. Lett. 75, 2560 (1999).

${ }^{28}$ J. M. Lavine, S. P. Sawan, Y. T. Shieh, and A. J. Bellezza, Appl. Phys. Lett. 62, 1099 (1993).
${ }^{29}$ L. Tsybeskov, Ju. V. Vandyshev, and P. M. Fauchet, Phys. Rev. B 49, 7821 (1994).

${ }^{30}$ J. M. Rehm, G. L. Mclendon, L. Tsybeskov, and P. M. Fauchet, Appl. Phys. Lett. 66, 3669 (1995).

${ }^{31}$ C. G. Van de Walle and W. B. Jackson, Appl. Phys. Lett. 69, 2441 (1996).

${ }^{32}$ A. Shih, S. C. Lee, and C. T. Chia, Appl. Phys. Lett. 74, 3347 (1999).

${ }^{33}$ R. Tsu, H. Shen, and M. Dutta, Appl. Phys. Lett. 60, 112 (1991).

34 Colvard, C. T. A. Gant, M. V. Klein, R. Merlin, R. Fischer, H. Morkoc, and A. C. Gossard, Phys. Rev. B 31, 2080 (1985). 